\title{
RETRACTED CHAPTER: A Study on Email Security Through Cryptographic Techniques
}

\author{
Akhilesh Kumar Singh ${ }^{(凶)}$, Vinesh Kumar, and Sharad Pratap Singh \\ GLAU, Mathura, UP, India \\ \{akhileshkr. singh, vinesh. kumar, \\ sharad.singh\}@gla.ac.in
}

The original version of this chapt was tetracted: This chapter has been retracted by the Editors because it reports research adertaken by Shafiya Afzal Sheikh and M Tariq Banday. The retraction note this chapter is available at https://doi.org/10.1007/9783-030-37051-0_108 